\title{
Hypothesis-test-based landcover change detection using multi-temporal satellite images - A comparative study
}

\author{
S.P. Teng ${ }^{\text {a }}$, Y.K. Chen ${ }^{\text {b }}$, K.S. Cheng ${ }^{\text {c,* }}$, H.C. Lo ${ }^{\text {a }}$ \\ ${ }^{a}$ School of Forestry and Resource Conservation, National Taiwan University, Taipei, Taiwan, ROC \\ ${ }^{\mathrm{b}}$ School of Tourism, Ming-Chuan University, Taoyuan County, Taiwan, ROC \\ ${ }^{\mathrm{c}}$ Department of Bioenvironmental Systems Engineering, National Taiwan University, Taipei, Taiwan, ROC
}

Received 20 October 2006; received in revised form 16 June 2007; accepted 25 June 2007

\begin{abstract}
Remote sensing images and technologies have been widely applied to environmental monitoring, in particular landuse/landcover classification and change detection. However, the uncertainties involved in such applications have not been fully addressed. In this paper two hypothesis-test-based change detection methods, namely the bivariate joint distribution method and the conditional distribution method, are proposed to tackle the uncertainties in change detection by making decisions based on the desired level of significance. Both methods require a data set of class-dependent no-change pixels to form the basis for class-dependent hypothesis test. Using an exemplar study area in central Taiwan, performance of the proposed methods are shown to be significantly superior to two other commonly applied methods (the post-classification comparison and the image differencing methods) in terms of the overall change detection accuracies. The conditional distribution method takes into consideration the correlation between digital numbers of the pre- and post-images and the effect of the known pre-image digital number on the range of the post-image digital number, and therefore yields the highest change detection accuracy. It is also demonstrated that the class-dependent change detection is crucial for accurate landuse/landcover change detection. (C) 2008 Published by Elsevier Ltd on behalf of COSPAR.
\end{abstract}

Keywords: Landuse/landcover change detection; Hypothesis test; Remote sensing; Image differencing

\section{Introduction}

Landuse/landcover (LULC) change detection using remotely sensed images has been widely applied for environmental monitoring. Many methods of change detection have been proposed including post-classification comparison, temporal image differencing, temporal image rationing, image regression, multifractal analysis, principal component analysis, change vector analysis, and multivariate alteration detection (Nielsen et al., 1998). Lunetta and Elvidge (1999) and Deer (1995) gave thorough reviews for change detection techniques. All change detection methods involve usage of multi-temporal images that are acquired in different dates and have dif-

\footnotetext{
${ }^{*}$ Corresponding author.

E-mail address: rslab@ntu.edu.tw (K.S. Cheng).
}

ferent view angles, sun angles, atmospheric conditions, and spatial coverages. Therefore, prior to the implementation of change detection algorithms, radiometric, and geometric corrections need to be conducted to reduce errors in change detection. Radiometric correction can be done by methods such as the dark object subtraction (DOS), image regression, and other more sophisticated methods which may require measurements of atmospheric optical depth (Song et al., 2001; Deer, 1995). The effect of misregistration on change detection has been addressed by Swain et al. (1982) and Townshend et al. (1992). In addition, among all change detection methods the image differencing method (including differencing on raw images and transformed images like NDVI) is most widely applied (Weismiller et al., 1977; Brera and Shahrokhi, 1978; Coiner, 1980; Jensen and Toll, 1982; Nelson, 1983; Singh, 1986; Vogelmann, 1988; Cheng et al., 2004). It generally uses a threshold 
for grey levels of the image used for change detection to identify pixels associated with LULC changes. The image used for change detection is a difference image derived from the raw or transformed multi-temporal images with its digital numbers reflective of LULC changes. The threshold value is usually chosen as multiples of the standard deviation of digital numbers of the difference image. Different threshold values have been suggested (Liu, 1983; Nelson, 1983; Ridd and Liu, 1998; Fung and LeDraw, 1988; Sohl, 1999) and no single threshold value can be universally applied. In addition, how much confidence can be expected for the results of change detection is seldom addressed except in rare cases where adequate validation data are available (Yuan and Elvidge, 1998). Therefore, the objectives of this paper are (1) to address some crucial but often neglected considerations in threshold determination and (2) to propose a hypothesis-test approach which provides a level of significance for the results of change detection.

\section{Study area and data}

A 7.3 - Richter scale earth quake occurred on September 21, 1999 in central Taiwan and drastically changed the landscape in extensive mountainous regions. After the earthquake, rocks, and soil piled on the hilly areas become unstable and are vulnerable to intensive storms. As a result, typhoon seasons of the following years have seen repeated occurrences of landslides and debris flows in central Taiwan. On July 2, 2004 the Mindulle Typhoon passed through Taiwan and brought intensive and huge amount of rainfall (over $1600 \mathrm{~mm}$ in three days) to mountainous regions of central Taiwan causing extensive landslides and severe debris flows. A mountainous area of approximately $250 \mathrm{~km}^{2}$ (see Fig. 1) is chosen for study of LULC changes caused by the Mindulle Typhoon. The study area is mostly covered by deciduous and coniferous forests and the water body of a major reservoir in central Taiwan. There are

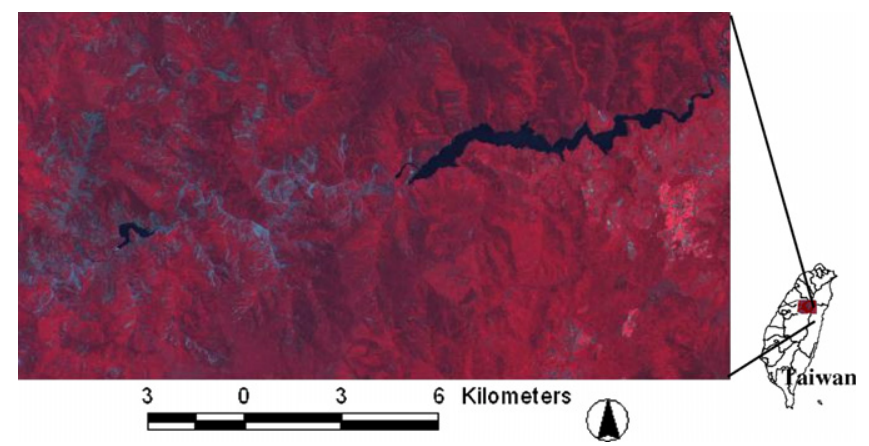

Fig. 1. The study area in central Taiwan. The false-color image is displayed using digital numbers of green (in blue), red (in green) and near infrared (in red) channels. (For interpretation of the references to color in this figure legend, the reader is referred to the web version of this paper.)

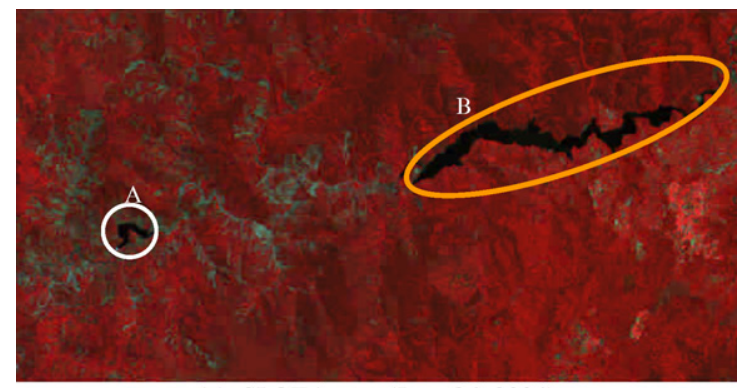

(a) SPOT image (June 26, 2004)

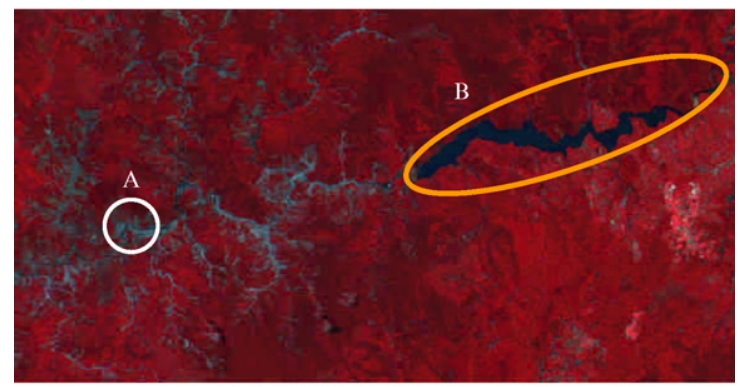

(b) SPOT image (July 12, 2004)

Fig. 2. The false-color pre- and post-images of the study area. Area $A$ was once a small land-locked lake (a) and the lake water was drained by a bank break (b). Area $B$ represents the reservoir pool. The false-color image is displayed the same way as in Fig. 1.

also scattered orchard plantations, grasslands, paved areas, and bare soils including landslide sites.

A set of orthorectified aerial photos at $1 / 5000$ scale taken soon after the typhoon event and SPOT-4 multispectral images (20 m spatial resolution) acquired immediately before (June 26) and after (July 12) the event were used in this study. The pre-event SPOT image was georeferenced using a set of ground control points chosen from the orthorectified aerial photos. Using a second order polynomial transformation, the root-mean-square (RMS) error of such image rectification was $1.7 \mathrm{~m}$. The post-event SPOT image was then registered to the pre-event image by image-toimage registration and achieved a RMS error of $0.15 \mathrm{~m}$. Hereafter the two SPOT images will be referred to as the pre- and post-images. Fig. 2 shows the false-color preand post-images.

\section{Methodologies}

The RMS errors of image registration were very small as compared to the pixel size. Therefore, the effect of misregistration on accuracy of change detection is considered to be minimal and will not be further discussed in this paper. Two hypothesis-test-based LULC change detection methods, namely the bivariate joint distribution method and the conditional distribution method, are proposed in this study and evaluation of their performance comparing to two other methods (the post-classification comparison and the image differencing methods) are conducted. The following subsections describe these methods and their procedures of change detection. 
3.1. Correction for the atmospheric effect using dark object subtraction

A fundamental premise of using remotely sensed images for change detection is that changes in objects of interest will result in changes in radiance values (or digital numbers) of corresponding pixels. However, the atmospheric condition and the sun-target-sensor geometry may vary from one image to the other, and therefore, pixels corresponding to the same object with no change occurrence may have different radiance values in different images. A few methods such as the dark object subtraction (DOS), also known as the histogram minimum method (Chavez, 1988; Cheng and Lei, 2001), and the relative radiometric normalization (Jenson, 1983; Song et al., 2001) have been applied for correction of the atmospheric effect.

The basic concept of DOS is to identify very dark features within the scene. Based on the principles of remote sensing, large, and clear water bodies and shadows cast by clouds or by large topographic features have near zero or very small digital numbers because clear water absorbs strongly and very little energy is scattered to the sensor from shadowed pixels. Therefore, the minimum scene radiance is set to be the upwelled radiance based on the assumption that it represents the radiance from a scene element with near zero reflectance. However, due to significant atmospheric scattering, values of the lowest digital number often are not at or near zero, but some larger values. Higher values of the lowest digital number are mainly contributed by the upwelled radiance. It is worthy to note that DOS only accounts for atmospheric scattering; the atmospheric absorption effect is not corrected. If an image has been processed by the dark object subtraction method, then its digital number is approximately a linear function of the earth surface reflectance.

The relative radiometric normalization techniques assume that digital values of the corresponding pixels in multi-date images are linearly related. The linear relationship can be determined by a least squares regression. Each regression model contains an additive term (interception) that accounts for the difference in upwelled radiance between dates and a multiplicative term (slope) that accounts for the difference in detector calibration, sun-target-sensor geometry, and atmospheric transmittance between dates.

In this study the lowest digital numbers were assumed to represent the values contributed by upwelled radiances and were subtracted from the original digital values of

Table 1

Lowest digital numbers of the SPOT multispectral images used in this study

Spectral band Pre-image (June 26, 2004) $\quad$ Post-image (July 12, 2004)

\begin{tabular}{llllllll} 
& Green & Red & Near IR & & Green & Red & Near IR \\
\hline Lowest DN & 25 & 7 & 0 & 21 & 8 & 0 \\
\hline
\end{tabular}

the satellite images. Table 1 illustrates the lowest digital numbers of the pre- and post-images. It can be seen clearly that the lowest digital numbers decrease with the increase of spectral wavelength since the effect of Rayleigh scattering decreases as the electromagnetic wavelength increases. The DOS-processed images were then used in subsequent analyses for each of the four change detection methods.

\subsection{Post-classification comparison}

This method of LULC change detection involves two major steps - (1) independently classifying multi-date images, and (2) comparing the LULC class memberships of the corresponding pixels in the multi-date images. Pixel pairs with different class labels in multi-date images are identified as change pixels. The accuracy of LULC classification depends on several inter-related factors including the number and types of image features used for classification, the desired level of classification, and the level of sophistication of the classification algorithm. If higher levels of LULC classification are desired, for example Anderson Level-II and Level-III LULC classes (Anderson et al., 1976), more bands of images and a more sophisticated classification algorithm may be required. However, limited by the number of available spectral bands of satellite images, the classification accuracy generally decreases with the increase of the desired level of LULC classes. In this study, only three major LULC classes - vegetation, water body and barren land, are specified in order to achieve high classification accuracy for each of the multi-date images. The maximum likelihood classification was implemented using all of the three spectral bands (green, red, and near infrared) and a set of training data in the pre- and post-images. The confusion matrices of the pre- and post-images LULC classification are shown in Table 2. Very high overall classification accuracies were achieved for both the pre- and the post-images. Also, all producer's and user's accuracies are high with the only exception of the user's accuracy of the barren land class of the Preimage classification. It is worthy to note that with the three major LULC classes, minor within-class changes, for example changes due to vegetation growth, soil moisture variation, etc., will likely not be detected.

LULC change detection was then conducted by comparing the class labels of all pixel pairs within the study area. Table 3 summarizes the results of LULC change detection. The overall percentage of detected changes is $11.19 \%$. Changes in the pre-image vegetation cover accounts for approximately $10 \%$ of the total study area. Even though changes in the pre-image water body only accounts for $0.23 \%$ of the study area, however, it accounts for approximately $14.5 \%[1462 /(1462+8615) \times 100 \%=14.51 \%]$ of the pre-image water body. Fig. 3 demonstrates the results of change detection using the post-classification comparison method. 
Table 2

Confusion matrices of the pre- and post-images LULC classification

\begin{tabular}{|c|c|c|c|c|c|c|}
\hline & \multirow[t]{2}{*}{ Class } & \multicolumn{3}{|c|}{ Ground truth } & \multirow[t]{2}{*}{ Sum } & \multirow[t]{2}{*}{ User's accuracy } \\
\hline & & WB & $\mathrm{V}$ & $\mathrm{BL}$ & & \\
\hline \multicolumn{7}{|c|}{ Pre-image classification } \\
\hline & $\mathrm{V}$ & 0 & 20,481 & 0 & 20,481 & $100.00 \%$ \\
\hline & BL & 199 & 818 & 2852 & 3869 & $73.71 \%$ \\
\hline & Sum & 1122 & 21,299 & 2998 & 25,319 & Overall Accuracy $95.80 \%$ \\
\hline \multirow[t]{5}{*}{ Classified } & WB & 1014 & 0 & 35 & 1049 & $96.66 \%$ \\
\hline & $\mathrm{V}$ & 0 & 20,849 & 31 & 20,880 & $99.85 \%$ \\
\hline & $\mathrm{BL}$ & 35 & 48 & 3504 & 3588 & $97.66 \%$ \\
\hline & Sum & 1050 & 20,897 & 3570 & 25,517 & Overall accuracy $99.41 \%$ \\
\hline & Producer's accuracy & $96.57 \%$ & $99.77 \%$ & $98.15 \%$ & $\kappa$ coefficient 0.98 & \\
\hline
\end{tabular}

WB, water body; V, vegetation; BL, barren land.

Table 3

Results of LULC change detection by the post-classification comparison method

\begin{tabular}{llll}
\hline $\begin{array}{l}\text { Pre-image } \\
\text { LULC class }\end{array}$ & $\begin{array}{l}\text { Number of no- } \\
\text { change pixels }\end{array}$ & $\begin{array}{l}\text { Number of } \\
\text { change pixels }\end{array}$ & $\begin{array}{l}\text { Percentage of } \\
\text { changes }\end{array}$ \\
\hline WB & 8615 & 1462 & $0.23^{*}$ \\
V & 490,573 & 65,157 & 10.41 \\
BL & 56,630 & 3463 & 0.55 \\
Sum & 555,818 & 70,082 & 11.19 \\
Total number of pixels within the study area: 625,900 & \\
${ }^{*}(1462 / 625,900) \times 100 \%=0.23 \%$ &
\end{tabular}

\subsection{Image differencing using NDVI}

The image differencing method is a technique whereby changes in brightness values between two or more data sets are determined by pixel-by-pixel subtraction of coregistered image data sets. The subtraction (differencing) produces an image data set where positive and negative values represent areas of change and values close to zero indicate areas that remain relatively unchanged (Muchoney and Haack, 1994). Threshold values are often defined and used to indicate whether significant or relevant change has occurred. In a change detection practice using image differencing method, the first step is to determine the feature images from which the difference image is derived. The feature images are a pair of pre- and post-images with their digital numbers reflective of LULC changes. They can be the raw satellite images or transformed images, for example NDVI or principal component images. The choice of the feature images should consider not only their capability of reflecting changes but also the probability distribution of the resultant difference image, if the threshold is to have a sound, statistical basis. Digital numbers in the difference image are often considered to be normally distributed with a zero mean and some value of standard deviation. In this study, the NDVI images are chosen as the feature images for two reasons: (1) the study area is mostly covered by vegetation canopy and, for change detection purpose, the difference between NDVI of multi-date images should give a reasonable indication of change in the vegetation canopy; (2) digital numbers of the NDVI images often are approximately normally distributed.

The pre- and post-NDVI images were derived using Eq. (1) and the NDVI difference image ( $\triangle$ NDVI) was created using Eq. (2).

$$
\begin{aligned}
& \mathrm{NDVI}=(\mathrm{NIR}-R) /(\mathrm{NIR}+R) \\
& \Delta \mathrm{NDVI}=\mathrm{NDVI}_{\mathrm{pre}}-\mathrm{NDVI}_{\mathrm{post}}
\end{aligned}
$$

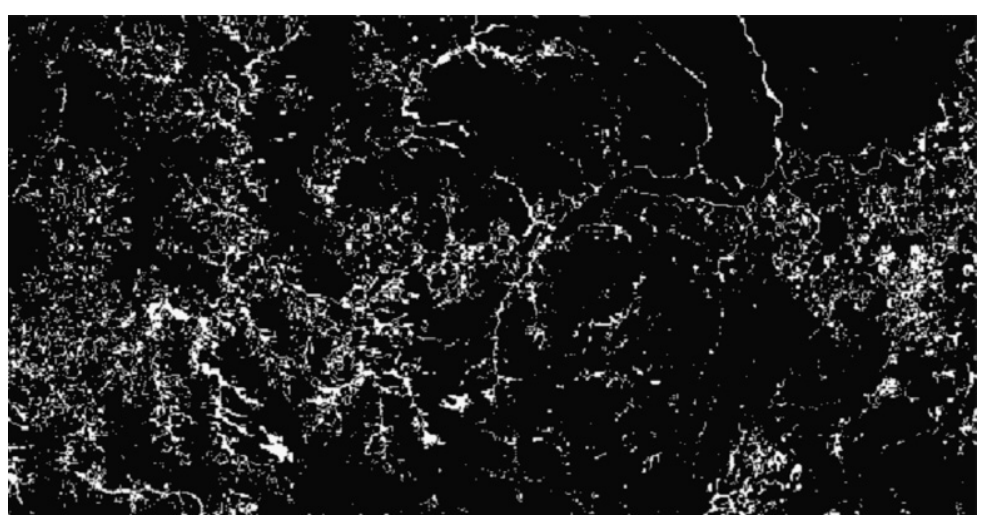

Fig. 3. Results of change detection by the post-classification comparison method. Areas with change occurrences are shown in white color. 


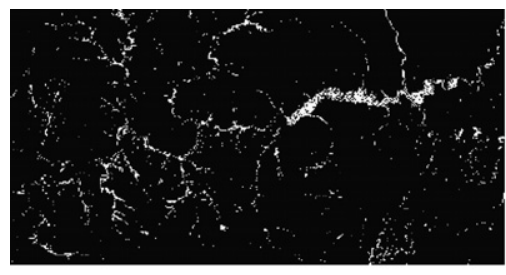

Threshold: $2.575 \sigma_{\triangle N D V I}$

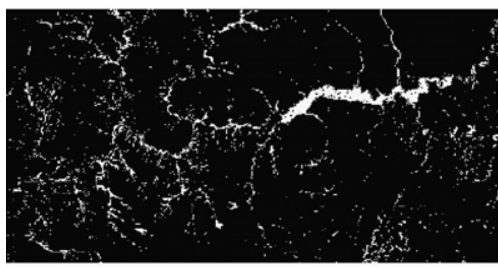

Threshold: $1.96 \sigma_{\triangle N D V I}$

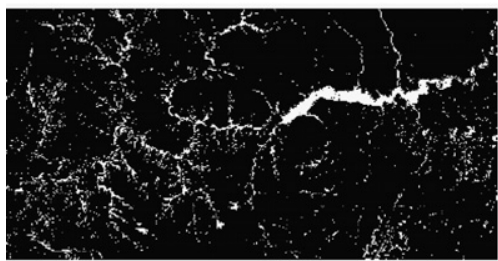

Threshold: $1.645 \sigma_{\Delta N D V I}$

(a) NDVI image differencing

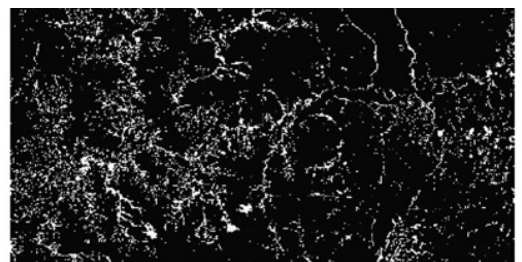

$\alpha=0.01$ (corresponding to

$2.575 \sigma_{\triangle N D V I}$ in column (a))

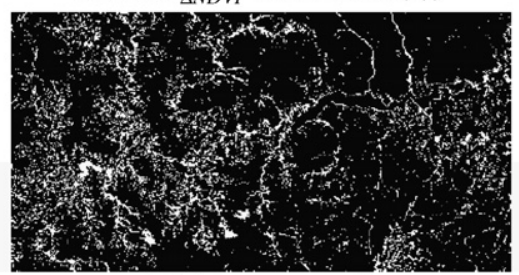

$\alpha=0.05$ (corresponding to

$1.96 \sigma_{\Delta N D V I}$ in column (a))

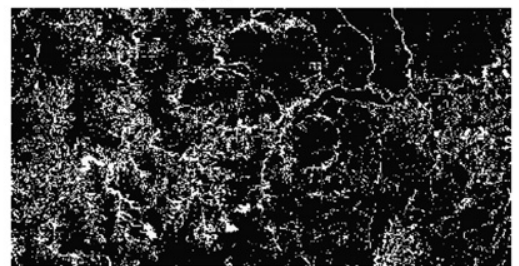

$\alpha=0.1$ (corresponding to

$1.645 \sigma_{\Delta N D V I}$ in column (a))

(b) NDVI bivariate joint distribution

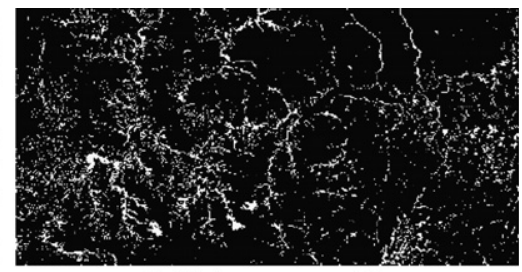

$\alpha=0.01$ (corresponding to

$2.575 \sigma_{\Delta N D V I}$ in column (a))

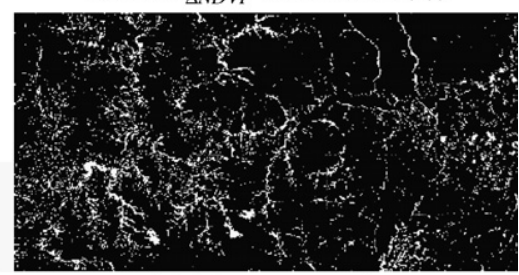

$\alpha=0.05$ (corresponding to

$1.96 \sigma_{\Delta N D V I}$ in column (a))

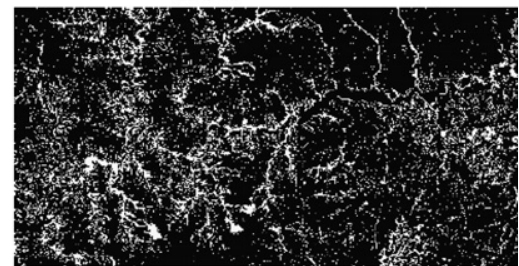

$\alpha=0.1$ (corresponding to

$1.645 \sigma_{\triangle N D V I}$ in column (a))

(c) NDVI conditional distribution

Fig. 4. Results of change detection by (a) the image differencing method, (b) the bivariate joint distribution method, and (c) the conditional distribution method. Areas with change occurrences are shown in white color.

where NIR and $R$, respectively, represent the digital numbers of the near infrared and red channel images. Values of $\triangle$ NDVI are assumed to be normally distributed with a zero mean and standard deviation $\sigma_{\Delta \mathrm{NDVI}}$, and pixels with absolute value of $\Delta$ NDVI greater than a selected threshold value are identified as change pixels. The threshold value is usually chosen as multiple of the standard deviation, i.e. $k \sigma_{\Delta \mathrm{NDVI}}$. In this study, several values of $k(1.645,1.96$, and 2.575$)$ corresponding to various exceedance probabilities $(0.1,0.05$, and 0.01$)$ were used for LULC change detection. The results of change detection by the NDVI image differencing method using different threshold values are shown in Fig. 4(a).

\subsection{Bivariate joint distribution method}

As implied by the relative radiometric normalization technique, digital numbers of the corresponding no-change pixels in multi-date images are linearly related due to difference in upwelled radiance, sun-target-sensor geometry, and atmospheric transmittance between dates. The linear relationship is not deterministic and the distribution cloud of digital numbers is usually characterized by a least square regression. Therefore, it is intuitive to consider using the joint probability distribution of the digital numbers of the pre- and post-images for change detection.
Let the digital numbers of the pre- and post-images be respectively represented by random variables $X_{1}$ and $X_{2}$. Assume that the two random variables form a bivariate normal (BVN) distribution and their joint probability density function can be expressed by the following equation:

$\phi(X)=\frac{1}{2 \pi|\Sigma|^{1 / 2}} \mathrm{e}^{-\frac{1}{2}\left[(X-\mu)^{\prime} \sum^{-1}(X-\mu)\right]}$

where $X^{\mathrm{T}}=\left(\begin{array}{ll}X_{1} & X_{2}\end{array}\right)$ and $\mu$ and $\sum$ are, respectively, the mean vector and covariance matrix of $X_{1}$ and $X_{2}$. It can also be shown that $(X-\mu)^{\prime} \sum^{-1}(X-\mu)$ is a $\chi^{2}$ distributed univariate random variable with degree of freedom 2 . Therefore, the probability contours of the BVN distribution can be established by

$$
(X-\mu)^{\prime} \sum^{-1}(X-\mu)=c
$$

for any positive constant of $c$. For example, the 95\% probability contour corresponds to

$P\left[(X-\mu)^{\prime} \sum^{-1}(X-\mu) \leqslant \chi_{0.95,2}^{2}\right]=0.95$

and can be constructed by assigning $c=\chi_{0.95,2}^{2}$ in Eq. (4). Other probability contours can also be constructed in a similar manner.

The concept of change detection using the bivariate joint distribution is illustrated in Fig. 5. If there are no change 


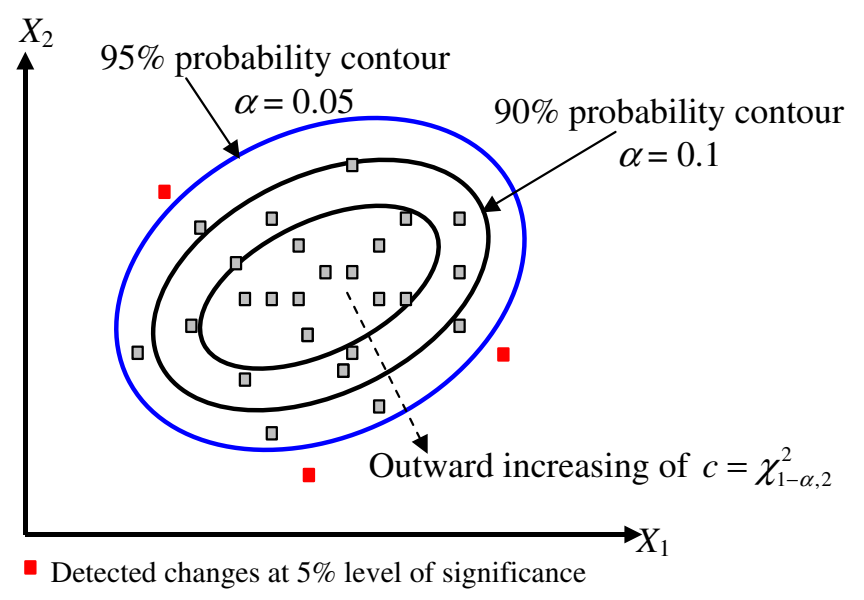

Fig. 5. Change detection using bivariate normal distribution.

occurrences in the study area, digital numbers of the corresponding pixels in the pre- and post-images should be correlated and can be characterized by a bivariate normal distribution. As $c$ in Eq. (4) increases, the probability for a no-change pixel pair to fall outside of the corresponding probability contour decreases. Therefore, from a hypothesis test point of view, the probability contour which corresponds to a given level of significance $\alpha$ (for example, the $95 \%$ probability contour corresponds to $\alpha=0.05$ level of significance) can be used to establish the critical region for the hypothesis test with null hypothesis $H_{\mathrm{o}}$ : no change, at the given level of significance $\alpha$. All pixel pairs fall outside of the $100(1-\alpha) \%$ probability contour are considered as change pixels. The bivariate joint distribution method takes into consideration the correlation between the digital numbers in the pre- and post-images. Such correlation is due to difference in upwelled radiance, sun-target-sensor geometry, and atmospheric transmittance between dates.

Meanwhile, a few important concerns must be addressed before the work of change detection can be placed in the framework of hypothesis test. Firstly, parameters (mean and covariance matrix) of the BVN distribution must be estimated using data of no-change pixel pairs only. This is due to the null hypothesis of no change occurrence. Secondly, the bivariate joint distribution may vary with LULC classes. For example, water body and vegetation canopy have quite different spectral reflectances, and as a result, the BVN distributions of the two classes are different. Therefore, for a given level of significance, the corresponding probability contour must be constructed for each LULC class.

In this study, we consider the BVN distribution of the pre- and post-NDVI images for change detection using the bivariate joint distribution method. The first step of this approach is to select a group of no-change pixel pairs for each LULC class. This can be achieved by using the change detection results of the post-classification comparison method. No-change pixel pairs for each of the three LULC classes were identified and formed the basis for estimation of $\mu$ and $\sum$ in Eq. (4) for each LULC class. Since the postclassification comparison method involves only three major
LULC classes and yields very high overall accuracies, the identified no-change pixels can be considered to be very reliable. Using the estimated values of $\mu$ and $\sum$ of each LULC class, $100(1-\alpha) \%$ probability contour can then be constructed for each of the three LULC classes. For any pixel pair in the study area, we first check its pre-image class identity based on the results of LULC classification, and then the $100(1-\alpha) \%$ robability contour of that class is used for change detection of the pixel pair. The results of change detection using the NDVI bivariate joint distribution method are shown in Fig. 4(b).

\subsection{Conditional distribution method}

At a given level of significance $\alpha$, the bivariate joint distribution method establishes the class-specific $100(1-\alpha) \%$ probability contours using all no-change pixel pairs of the corresponding LULC classes. However, given the digital number in the pre-image, the digital number in the postimage is likely to be restricted to fall within a smaller range, if no change has occurred. As illustrated in Fig. 6, if a nochange pixel pair has its pre-image digital number equals $x_{1}$, then; its post-image digital number is more likely to fall closer to $b$, and very unlikely to be close to the value $a$. Knowing the pre-image digital number provides us additional information for making decision on change detection. To take the advantage of knowing the pre-image digital number, we therefore propose to use the conditional distribution of the post-image digital number for change detection.

Assuming a BVN distribution for digital numbers of a no-change pixel pair, the conditional distribution of the post-image digital number $\left(X_{2}\right)$, given the pre-image digital number $\left(X_{1}=x_{1}\right)$, can be expressed as

$$
\begin{aligned}
& f\left(X_{2}=\right.\left.x_{2} \mid x_{1}\right)=\frac{1}{\sqrt{2 \pi\left(1-\rho^{2}\right)} \cdot \sigma_{2}} \\
& \cdot \exp \left\{-\frac{1}{2}\left[\frac{\left(x_{2}-\mu_{2}\right)-\rho \frac{\sigma_{2}}{\sigma_{1}}\left(x_{1}-\mu_{1}\right)}{\sigma_{2} \sqrt{1-\rho^{2}}}\right]^{2}\right\}
\end{aligned}
$$

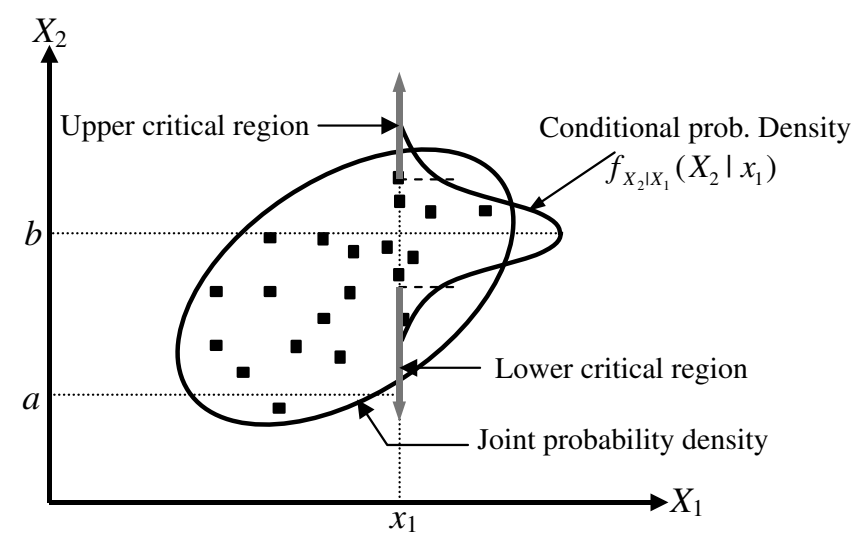

Fig. 6. Change detection using conditional distribution method. 
where $\mu_{i}$ and $\sigma_{i}(i=1,2)$ are, respectively, the mean and standard deviation of $X_{i}$, and $\rho$ is the correlation coefficient between $X_{1}$ and $X_{2}$. Similar to the bivariate joint distribution method, these parameters $\left(\mu_{i}, \sigma_{i}\right.$, and $\left.\rho\right)$ are LULC class-dependent and must be estimated using only the nochange pixel pairs of individual LULC classes.

The conditional distribution of the post-image digital number is also a normal distribution with mean and standard deviation, respectively, equal to $\mu_{2}+\rho \frac{\sigma_{2}}{\sigma}\left(x_{1}-\mu_{1}\right)$ and $\sigma_{2} \sqrt{1-\rho^{2}}$. Thus, using the standard normal distribution, the decision of the hypothesis test $H_{\mathrm{o}}$ : no change, given $X_{1}=x_{1}$ and at the level of significance $\alpha$, can be made by rejecting $H_{\mathrm{o}}$, if

$$
\begin{aligned}
& {\left[\mu_{2}+\rho \frac{\sigma_{2}}{\sigma_{1}}\left(x_{1}-\mu_{1}\right)\right]-k_{\alpha}\left(\sigma_{2} \sqrt{1-\rho^{2}}\right)} \\
& \quad>x_{2} \text { or }\left[\mu_{2}+\rho \frac{\sigma_{2}}{\sigma_{1}}\left(x_{1}-\mu_{1}\right)\right]+k_{\alpha}\left(\sigma_{2} \sqrt{1-\rho^{2}}\right)<x_{2}
\end{aligned}
$$

In the above inequalities, the choice of $k_{\alpha}$ is dependent on the desired level of significance $\alpha$. For $\alpha$ values of $0.01,0.05$, and 0.1 , the corresponding values of $k_{\alpha}$ are $2.575,1.96$, and 1.645 , respectively.

As illustrated in Fig. 7, unlike the bivariate joint distribution method, the critical region of the conditional distribution method is determined by two parallel lines. Note that, at $X_{1}=x_{1}$, the point marked by $\boldsymbol{\Delta}$ is identified as a change pixel by the conditional distribution method and a nochange pixel by the bivariate joint distribution method. On the contrary, the point marked by is identified as a nochange pixel by the conditional distribution method and a change pixel by the bivariate joint distribution method. It is worthy to reiterate that the parallel lines defining the critical region of the hypothesis test are LULC class dependent.
Again, the pre- and post-NDVI images were used for change detection using the conditional probability distribution method. For any pixel pair in the study area, the preimage class identity was firstly checked based on the results of LULC classification, and then critical region, corresponding to the identified LULC class, of the post-image NDVI value is used for change detection of the pixel pair. The results of change detection using the conditional distribution method are shown in Fig. 4(c).

\section{Performance comparison of different change detection methods}

Upon a visual inspection of the pre- and post-images in Fig. 2, a few significant and extensive change and nochange areas can be easily identified. For example, the area $A$ was once a small land-locked lake formed by landslides on steep river banks and the lake water was drained by a bank break induced by the high streamflow during the Mindulle Typhoon period. In contrast, the reservoir pool in area $B$ remained unchanged, even though the suspended sediment concentration increased after the typhoon event, causing higher spectral reflectance in the post-image. The NDVI image differencing method failed to detect changes in area $A$ and identified pixels in the water pool in area $B$ as change pixels. All other methods successfully detected change and no-change occurrences in area $A$ and $B$, respectively. Such results can be attributed to (1) thresholds in the image differencing method are not LULC-class dependent, and therefore, the threshold values are overly influenced by the dominant LULC class (vegetation cover in our study), and (2) both the hypothesis-test based bivariate joint distribution and conditional distribution methods consider the LULC-class-dependent correlation between NDVI values of the pre- and post-images.

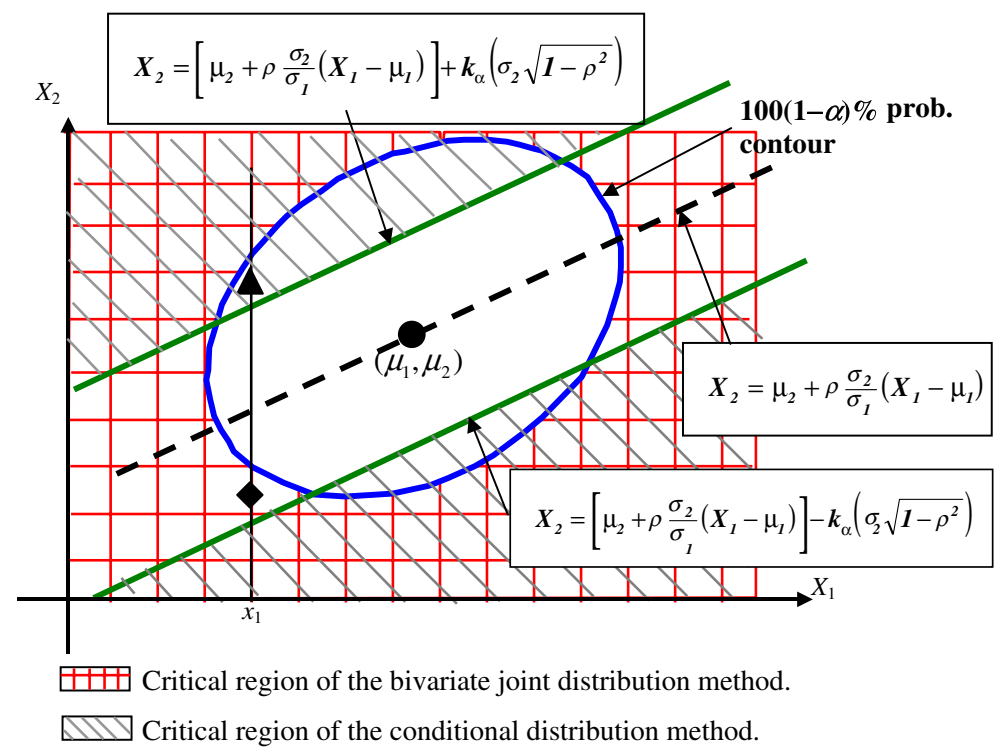

Fig. 7. Comparison of the critical regions of the bivariate joint distribution and the conditional distribution methods. 
In addition to the above rough evaluation, a more thorough comparison was conducted using a set of carefully selected validation data which includes a total of 8392 pixels (3575 change pixels and 4817 no-change pixels), accounting for $1.34 \%$ of the total number of pixels in the study area. The validation data were selected using two sets of high spatial resolution orthorectified aerial photos taken before and after the typhoon event and based on several trips of field investigation. Performance comparison for all change detection methods was conducted by adopting the concept of confusion matrix in image classification using the validation data (see Tables 4-7). The two hypothesis-test-based methods are significantly superior to the post-classification comparison and the image differencing methods in terms of the overall accuracy and the $\kappa$ coefficient. Both the bivariate joint distribution method and the conditional distribution method consider the correlation between digital numbers of no-change pixels in the pre- and post-images, and therefore, the effect of sun-target-sensor geometry and the atmospheric transmittance on radiances of multi-date images are implicitly accounted for. The conditional distribution method further considers the effect of the known pre-image digital number on the variation range of the post-image digital number and yields slightly better change detection accuracies.

Table 8 shows the percentages of change pixels identified by different methods, under different levels of significance. Combining the information contained in Tables 4-7 and
Table 8 , it can be seen that both the percentage of identified change pixels and the overall accuracy tend to increase with the increase of the level of significance $\alpha$. Such results are reasonable since the heavy rainfall brought by the Mindulle Typhoon induced extensive and widespread landslides and debris flows in the study area, resulted in high percentage of LULC changes. In comparison, from the results of another change detection study (Chung, 2006) carried out in an area with very few LULC changes, we found that both the percentage of identified change pixels and the overall accuracy increase with the decrease of the level of significance $\alpha$.

Similar to the two hypothesis-test-based methods, the image differencing method also choose multiples of the standard deviation as the thresholds for change detection. However, the standard deviation is estimated using all pixels in the image, including change and no-change pixels, and therefore, its change detection accuracies cannot be evaluated with different levels of significance. The accuracy of change detection achieved by the postclassification comparison method also cannot be evaluated with the level of significance since the decision on change detection does not consider the probability distribution of digital numbers of the involved images and no threshold or critical regions are required. However, the method of post-classification comparison yields better change detection accuracy than the image differencing method.

Table 4

Validation of change detection - post-classification comparison method

\begin{tabular}{|c|c|c|c|c|c|}
\hline & \multirow[t]{2}{*}{ Class } & \multicolumn{2}{|c|}{ Ground truth } & \multirow[t]{2}{*}{ Sum } & \multirow[t]{2}{*}{ User's accuracy } \\
\hline & & Change & No change & & \\
\hline \multirow[t]{3}{*}{ Identified } & Change & 2703 & 161 & 2864 & $94.38 \%$ \\
\hline & Sum & 3575 & 4817 & 8392 & Overall accuracy $87.69 \%$ \\
\hline & Producer's accuracy & $75.61 \%$ & $96.66 \%$ & $\kappa$ coefficient 0.74 & \\
\hline
\end{tabular}

Table 5

Validation of change detection - NDVI image differencing method

\begin{tabular}{|c|c|c|c|c|c|}
\hline & \multirow[t]{2}{*}{ Class } & \multicolumn{2}{|c|}{ Ground truth } & \multirow[t]{2}{*}{ Sum } & \multirow[t]{2}{*}{ User's accuracy } \\
\hline & & Change & No change & & \\
\hline \multicolumn{6}{|c|}{ (a) Threshold: $2.575 \sigma_{\Delta N D V I}$} \\
\hline \multirow[t]{4}{*}{ Identified } & Change & 2014 & 275 & 2296 & $87.99 \%$ \\
\hline & No change & 1561 & 4542 & 6096 & $74.42 \%$ \\
\hline & Sum & 3575 & 4817 & 8392 & Overall accuracy $78.12 \%$ \\
\hline & Producer's accuracy & $56.34 \%$ & $94.29 \%$ & $\kappa$ coefficient 0.53 & \\
\hline \multicolumn{6}{|c|}{ (b) Threshold: $1.96 \sigma_{\Delta N D V I}$} \\
\hline \multirow[t]{4}{*}{ Identified } & Change & 2463 & 371 & 2834 & $86.91 \%$ \\
\hline & No change & 1112 & 4446 & 5558 & $79.99 \%$ \\
\hline & Sum & 3575 & 4817 & 8392 & Overall accuracy $82.33 \%$ \\
\hline & Producer's accuracy & $68.90 \%$ & $92.30 \%$ & $\kappa$ coefficient 0.63 & \\
\hline \multicolumn{6}{|c|}{ (c) Threshold: $1.645 \sigma_{\Delta N D V I}$} \\
\hline \multirow[t]{4}{*}{ Identified } & Change & 2649 & 399 & 3048 & $86.91 \%$ \\
\hline & No change & 926 & 4418 & 5344 & $82.67 \%$ \\
\hline & Sum & 3575 & 4817 & 8392 & Overall accuracy $84.21 \%$ \\
\hline & Producer's accuracy & $74.10 \%$ & $91.72 \%$ & $\kappa$ coefficient 0.67 & \\
\hline
\end{tabular}


Table 6

Validation of change detection - Bivariate joint distribution method

\begin{tabular}{|c|c|c|c|c|c|}
\hline & \multirow[t]{2}{*}{ Class } & \multicolumn{2}{|c|}{ Ground truth } & \multirow[t]{2}{*}{ Sum } & \multirow[t]{2}{*}{ User's accuracy } \\
\hline & & Change & No change & & \\
\hline \multicolumn{6}{|l|}{ (a) $\alpha=0.01$} \\
\hline \multirow[t]{4}{*}{ Identified } & Change & 2968 & 52 & 3020 & $98.28 \%$ \\
\hline & No change & 607 & 4765 & 5372 & $88.70 \%$ \\
\hline & Sum & 3575 & 4817 & 8392 & Overall accuracy $92.15 \%$ \\
\hline & Producer's accuracy & $83.02 \%$ & $98.92 \%$ & $\kappa$ coefficient 0.84 & \\
\hline \multicolumn{6}{|l|}{ (b) $\alpha=0.05$} \\
\hline \multirow[t]{4}{*}{ Identified } & Change & 3132 & 112 & 3244 & $96.55 \%$ \\
\hline & No change & 443 & 4705 & 5148 & $91.39 \%$ \\
\hline & Sum & 3575 & 4817 & 8392 & Overall accuracy $93.39 \%$ \\
\hline & Producer's accuracy & $87.61 \%$ & $97.67 \%$ & $\kappa$ coefficient 0.86 & \\
\hline \multicolumn{6}{|l|}{ (c) $\alpha=0.1$} \\
\hline \multirow[t]{4}{*}{ Identified } & Change & 3233 & 302 & 3535 & $91.46 \%$ \\
\hline & No change & 342 & 4515 & 4857 & $92.96 \%$ \\
\hline & Sum & 3575 & 4817 & 8392 & Overall accuracy $92.33 \%$ \\
\hline & Producer's accuracy & $90.43 \%$ & $93.73 \%$ & $\kappa$ coefficient 0.84 & \\
\hline
\end{tabular}

Table 7

Validation of change detection - conditional distribution method

\begin{tabular}{|c|c|c|c|c|c|}
\hline & \multirow[t]{2}{*}{ Class } & \multicolumn{2}{|c|}{ Ground truth } & \multirow[t]{2}{*}{ Sum } & \multirow[t]{2}{*}{ User's accuracy } \\
\hline & & Change & No change & & \\
\hline \multicolumn{6}{|c|}{ (a) $\alpha=0.01$} \\
\hline \multirow[t]{4}{*}{ Identified } & Change & 3006 & 52 & 3058 & $98.30 \%$ \\
\hline & No change & 569 & 4765 & 5334 & $89.33 \%$ \\
\hline & Sum & 3575 & 4817 & 8392 & Overall accuracy $92.60 \%$ \\
\hline & Producer's accuracy & $84.08 \%$ & $98.92 \%$ & $\kappa$ coefficient 0.85 & \\
\hline \multicolumn{6}{|c|}{ (b) $\alpha=0.05$} \\
\hline \multirow[t]{4}{*}{ Identified } & Change & 3177 & 113 & 3290 & $96.57 \%$ \\
\hline & No change & 398 & 4704 & 5102 & $92.20 \%$ \\
\hline & Sum & 3575 & 4817 & 8392 & Overall accuracy $93.91 \%$ \\
\hline & Producer's accuracy & $87.87 \%$ & $97.65 \%$ & $\kappa$ coefficient 0.87 & \\
\hline \multicolumn{6}{|l|}{ (c) $\alpha=0.1$} \\
\hline \multirow[t]{4}{*}{ Identified } & Change & 32,993 & 186 & 3452 & $94.61 \%$ \\
\hline & No change & 309 & 4631 & 4940 & $93.74 \%$ \\
\hline & Sum & 3575 & 4817 & 8392 & Overall accuracy $94.10 \%$ \\
\hline & Producer's accuracy & $91.36 \%$ & $96.14 \%$ & $\kappa$ coefficient 0.88 & \\
\hline
\end{tabular}

Table 8

Percentage of the change pixels detected by different methods

\begin{tabular}{llccc}
\hline \multirow{2}{*}{ Level of significance $\alpha$} & \multicolumn{4}{l}{ Change detection method } \\
\cline { 2 - 5 } & Image differencing & Bivariate joint distribution & Conditional distribution & Post-classification comparison \\
\hline 0.01 & 3.81 & 9.90 & 9.58 & 11.19 \\
0.05 & 5.90 & 13.67 & 13.21 & 11.19 \\
0.1 & 7.30 & 17.02 & 16.61 & 11.19 \\
\hline
\end{tabular}

Note: For the image differencing method, $\alpha=0.01,0.05$ and 0.1 correspond to threshold of $2.575 \sigma_{\Delta \mathrm{NDVI}}, 1.96 \sigma_{\Delta \mathrm{NDVI}}$ and $1.645 \sigma_{\Delta \mathrm{NDVI}}$, respectively.

Placing the work of change detection in the framework of hypothesis test has the advantage that the uncertainties involved in change detection can be quantitatively evaluated through different levels of significance, as have been demonstrated by the bivariate joint distribution method and the conditional distribution method. This is achieved by using only the class-dependent, no-change pixel pairs, which can be identified by conducting LULC classification prior to the change detection, to establish critical regions for different LULC classes. The results of LULC change detection in this study suggest that a prior LULC classification can help to achieve independent change detection for individual LULC classes and the class-dependent change detection is crucial for accurate LULC change detection.

A final note about choosing the change detection feature is also worth addressing. The hypothesis-test-based method 
is based on the conditional probability density of a chosen change detection variable. Practically speaking, the change detection variable can be any variable which is sensitive to changes of conditions and properties of surface features. Although in our study we have chosen the NDVI images for change detection, there are other features that may be considered. Notably, FAPAR (the fraction of absorbed photosynthetically active radiation) images are more sensitive to conditions and properties of vegetation cover and also have minimum sensitivity to atmospheric scattering and absorption effects, soil color and brightness changes, and temporal and spatial variations in the geometry of illumination and observation. Therefore, application of the proposed hypothesis-test-based approach using FAPAR as the change detection feature is highly recommended.

\section{Conclusions}

Two hypothesis-test-based LULC change detection methods, namely the bivariate joint distribution and the conditional distribution methods, are proposed and compared with the post-classification comparison method and the image differencing method. Based on the change detection results in a study area in central Taiwan, the two proposed methods are significantly superior to the other two methods in terms of the overall change detection accuracy, with the conditional distribution method having the highest accuracies. Unlike the other three methods, the postclassification comparison method does not require a threshold for change detection. The threshold value of the image differencing method is determined using digital numbers of all pixels in the difference image, and therefore, its change detection accuracy cannot be evaluated with a level of significance. In contrast, the bivariate joint distribution method and the conditional distribution method are hypothesis-test based, with LULC-class-dependent critical regions which are associated with desired levels of significance. Both the bivariate joint distribution method and the conditional distribution method consider the correlation between digital numbers of no-change pixels in the pre- and post-images, and therefore, the effect of sun-target-sensor geometry and atmospheric transmittance on radiances of multi-date images is implicitly accounted for. The conditional distribution method further considers the effect of the known pre-image digital number on the variation range of the post-image digital number and yields slightly better change detection accuracies. A prior LULC classification can help to achieve independent change detection for individual LULC classes and the class-dependent change detection is crucial for accurate LULC change detection.

\section{Acknowledgements}

We are grateful for the National Science Council and Water Resources Agency of Taiwan, ROC for funding research projects which lead to the results presented in this paper. We also thank the Aerial Survey Office of the Council of Agriculture for providing aerial photos and allowing usage of their facilities. The corresponding author is also grateful for the National Science Council of Taiwan, ROC and the Kyoto University, Japan for providing financial and facility supports for his sabbatical leave at the Kyoto University during which this manuscript was prepared. Special thanks are also extended to Professor Mitsuno and Dr. Torii at the Kyoto University for having many fruitful discussions with the corresponding author. Our thanks also extend to two anonymous reviewers for their insightful and constructive comments.

\section{References}

Anderson, J.R., Hardy, E.E., Roach, J.T., Witmer, R.E. A land use and land cover classification system for use with remote sensor data. Geological Survey Professional Paper No. 964, U.S. Government Printing Office, Washington, DC, 28p, 1976.

Brera, A.M., Shahrokhi, F. Application of Landsat data to monitor desert spreading in the Sahara region. 12th International Symposium on Remote Sensing of Environment, Ann. Arbor., Michigan, 1978.

Chavez Jr., P.S. An improved dark-object subtraction technique for atmospheric scattering correction of multispectral data. Remote Sensing of Environment 24, 459-479, 1988.

Cheng, K.S., Lei, T.C. Reservoir trophic state evaluation using Landsat TM images. J. Am. Water Res. Assoc. 37 (5), 13211334, 2001.

Cheng, K.S., Wei, C., Chang, S.C. Locating landslides using multitemporal satellite images. Adv. Space Res. 33, 296-301, 2004.

Chung, C.W. Landuse/landcover change detection using two-stage remote sensing image classification. Master thesis, Department of Bioenvironmental Systems Engineering, National Taiwan University, 108p, 2006.

Coiner, J.C. Using Landsat to monitor changes in vegetation cover induced by desertification processes. 14th International Symposium on Remote Sensing of Environment, Ann Arbor, Michigan, 1980.

Deer, P. Digital change detection techniques in remote sensing. Technical Report, DSTO-TR-0169, Department of Defence, Australia, 52p, 1995.

Fung, T., LeDraw, E. The determination of optimal threshold levels for change detection using various accuracy indices. Photogram. Eng. Remote sensing 54 (10), 1449-1454, 1988.

Jensen, J.R., Toll, D.L. Detecting residential land use development at the urban fringe. Photogram. Eng. Remote Sensing 48, 629-643, 1982.

Jenson, J.R. Urban/suburban landuse analysis, in: Jenson, J.R. (Ed.), Manual of Remote Sensing. American Society of Photogrammetry, pp. 1571-1666, 1983.

Liu, J. Creating a method for optimal threshold determination for change detection in an urban environment. M.S. thesis, Department of Geography, University of Utah, 1983.

Lunetta, R.S., Elvidge, C.D. Remote Sensing Change Detection. Taylor \& Francis Ltd., London, p318, 1999.

Muchoney, D.M., Haack, B.N. Change detection for monitoring forest defoliation. Photogram. Eng. Remote Sensing 60, 1243-1251, 1994.

Nelson, R.F. Detecting forest canopy change due to insect activity using Landsat MSS. Photogram. Eng. Remote Sensing 49, 1303-1314, 1983.

Nielsen, A.A., Conradsen, K., Simpson, J.J. Multivariate alteration detection (MAD) and MAF postprocessing in multispectral, bitemporal image data: new approaches to change detection studies. Remote Sensing Environ. 64, 1-19, 1998.

Ridd, M.K., Liu, J.A. comparison of four algorithms for change detection in an urban environment. Remote Sensing Environ. 63, 95-100, 1998.

Singh, A. Change detection in the tropical forest environment of northeastern India using Landsat, in: Eden, M.J., Parry, J.T. (Eds.), 
Remote Sensing and Tropical Land Management. John Wiley \& Sons, Chichester, pp. 237-254, 1986.

Sohl, T.L. Change analysis in the United Arab Emirates: an investigation of techniques. Photogram. Eng. Remote Sensing 65, 475-484, 1999.

Song, C., Woodcock, C.E., Seto, K.C., et al. Classification and change detection using Landsat TM data: when and how to correct atmospheric effects. Remote Sensing Environ. 75, 230-244, 2001.

Swain, P.H., Vanderbilt, V.C., Jobusch, C.D. A quantitative applications-oriented evaluation of Thematic Mapper design specifications. IEEE Trans. Geosci. Remote Sensing GE-20, 370-377, 1982.
Townshend, J.R.G., Justice, C.O., Gurney, C., McManus, J. The impact of misregistration on change detection. IEEE Trans. Geosci. Remote Sensing 30 (5), 1054-1060, 1992.

Vogelmann, J.E. Detection of forest change in the Green Mountains of Vermont using multispectral scanner data. Int. J. Remote Sensing 9, 1187-1200, 1988.

Weismiller, R.A., Kristof, S.J., Scholtz, D.K., et al. Change detection in coastal zone environments. Photogram. Eng. Remote Sensing 43, 1533-1539, 1977.

Yuan, D., Elvidge, C. NALC land cover change detection pilot study: Washington D.C. area experiment. Remote Sensing of Environ. 66, 166-178, 1998. 